\title{
Relations between the Spectral Indices and Flux Densities of Eight Blazars
}

\author{
Yu-hai Yuan (iD) ${ }^{1,2}$ \\ ${ }^{1}$ Center for Astrophysics, Guangzhou University, Guangzhou, Guangdong 510006, China \\ ${ }^{2}$ Astronomy Science and Technology Research Laboratory of Department of Education of Guangdong Province, \\ Guangzhou 510006, China \\ Correspondence should be addressed to Yu-hai Yuan; yh_yuan@gzhu.edu.cn
}

Received 4 March 2019; Revised 7 April 2019; Accepted 22 April 2019; Published 2 May 2019

Academic Editor: Geza Kovacs

Copyright (C) 2019 Yu-hai Yuan. This is an open access article distributed under the Creative Commons Attribution License, which permits unrestricted use, distribution, and reproduction in any medium, provided the original work is properly cited.

\begin{abstract}
Relations between the flux densities $(F)$ and spectral indices $(\alpha)$ can help us analyze the emission process. In this paper, we choose 8 blazars $(0235+164,0430+052,1156+295,3 \mathrm{C} 345,1308+326,1413+135,3 \mathrm{C} 454.3$, and 1749+096) from the University of Michigan Radio Observatory (UMRAO) database to study the relations between the spectral indices $(\alpha)$ and flux densities at $14.5 \mathrm{GHz}\left(F_{14.5}\right)$. The main results are the following. (1) There are strong anticorrelations between $\alpha$ and $F_{14.5}$, with the correlation coefficient $r$ in the range from -0.33 to -0.87 . (2) The $\alpha-F_{14.5}$ distributions show elliptic appearance, which have been fitted by elliptic curves. (3) For most of the sources, the time intervals of $\alpha-F_{14.5}$ elliptic circle $\left(T_{c}\right)$ are consistent with the quasi-periodicities calculated by the averaged light curves and spectral variances $\left(P_{\alpha \bar{F}}\right)$.
\end{abstract}

\section{Introduction}

Blazars are a subclass of active galactic nuclei (AGNs). They show some extreme properties, such as violent optical variability, core dominance, superluminal motion, and so on $[1,2]$. Blazars can be divided into two subclasses, BL Lacerate objects (BL Lacs), and flat-spectrum radio quasars (FSRQs). BL Lacs are characterized by featureless optical spectra or weak emission lines [3]. Flat radio spectrum and broad optical emission lines are typical for FSRQs [1]. Generally, the division between the two subclasses is based on the equivalent width $(E W)$ of the optical broad emission lines; BL Lacs show $E W<5 \AA[1,4-6]$.

The time scale of optical variability is an important quantity which is often used to probe the physical processes in blazars. The time scales are in the range from minutes to years and can be divided into three types: intraday variability (IDV), short-term variability, and long-term variability.

Usually, the intraday variability and short-term variability are nonperiodic, but the long-term variability is quasiperiodic. The long-term optical variabilities of blazars have been discussed by many papers [3,7-18]. The explanation about the long-term variabilities can be the binary black-hole model, the thermal instability model and the perturbation model and so on [14].

For blazars, there are many papers discussing relationship between the spectrum and flux density $[16,19-26]$. Dai et al. (2009) [27] presented long-term BVRI observations and discussed the correlations between color index and brightness. Beaklini \& Abraham (2014) [28] used variability at $7 \mathrm{~mm}$ to find evidence of shocks and precession in the jet. Fan et al. (2014) [15] analyzed the relation between $V$-band flux density $\left(F_{V}\right)$ and spectral index $(\alpha)$ for two nearby quasars and found two different relations below and above $F_{V}=28$ $\mathrm{mJy}$. When $F_{V}<28 \mathrm{mJy}, F_{V}$, and $\alpha$ showed anticorrelation, when $F_{V}>28 \mathrm{mJy}, F_{V}$, and $\alpha$ showed positive correlation. Yuan \& Fan (2015) [29] found that, in 3C273 and 3C446, there was elliptic structure in the distributions between the flux densities and spectral indices. Wierzcholska et al. (2015) [22] analyzed the color-magnitude correlations of 30 blazars and observed the bluer-when-brighter behaviors. Carnerero et al. (2017) [23] pointed out that the flux densities and spectral variability can be compatible with jet models including at least two emitting regions that can change their orientation with respect to the line of sight. Isler et al. (2017) [24] provided an explanation of the long-term optical-infrared 
TABLE 1: The spectral indices of 8 blazars.

\begin{tabular}{|c|c|c|c|c|c|c|}
\hline $\begin{array}{l}\text { Name } \\
\text { (1) }\end{array}$ & $\begin{array}{c}z \\
(2)\end{array}$ & $\begin{array}{c}\text { Type } \\
(3)\end{array}$ & $\begin{array}{l}N \\
(4)\end{array}$ & $\begin{array}{c}\alpha_{\min } \\
(5)\end{array}$ & $\begin{array}{c}\alpha_{\max } \\
(6)\end{array}$ & $\begin{array}{c}\bar{\alpha} \\
(7)\end{array}$ \\
\hline $0235+164$ & 0.94 & $B L$ & 129 & $-0.798 \pm 0.001$ & $0.463 \pm 0.012$ & $-0.082 \pm 0.279$ \\
\hline $0430+052$ & 0.033 & $F$ & 116 & $-0.384 \pm 0.001$ & $0.469 \pm 0.018$ & $0.127 \pm 0.200$ \\
\hline $1156+295$ & 0.7247 & $F$ & 66 & $-0.779 \pm 0.023$ & $0.264 \pm 0.006$ & $-0.221 \pm 0.249$ \\
\hline $1308+326$ & 0.998 & $B L$ & 128 & $-0.713 \pm 0.017$ & $0.231 \pm 0.012$ & $-0.283 \pm 0.236$ \\
\hline $1413+135$ & 0.2467 & $B L$ & 56 & $-1.098 \pm 0.001$ & $-0.183 \pm 0.038$ & $-0.583 \pm 0.260$ \\
\hline 3C 345 & 0.593 & $F$ & 187 & $-0.733 \pm 0.013$ & $0.296 \pm 0.024$ & $-0.136 \pm 0.230$ \\
\hline $1749+096$ & 0.322 & $B L$ & 125 & $-1.213 \pm 0.023$ & $-0.008 \pm 0.001$ & $-0.503 \pm 0.266$ \\
\hline 3C 454.3 & 0.859 & $F$ & 162 & $-0.339 \pm 0.038$ & $0.517 \pm 0.013$ & $0.171 \pm 0.217$ \\
\hline
\end{tabular}

color variabilities in some blazars and presented a general scheme which can apply to these variabilities.

The spectral indices are associated with the emission properties, and the flux densities demonstrate the optical variability properties. The study about the relations between the spectral indices and flux densities can combine the emission properties and optical variabilities. Generally, for BL Lacs, when the sources become brighter, the spectrum becomes flatter and when the sources become fainter, the spectrum becomes steeper.

This paper is arranged as follows. In Section 2, we calculate the spectral indices; in Section 3, we analyze the relations between the radio spectral index $\alpha$ and the 14.5 $\mathrm{GHz}$ radio flux density $F_{14.5}$; in Sections 4 and 5, we give the discussion and conclusions, respectively.

\section{The Spectral Indices}

Based on the UMRAO (University of Michigan Radio Astronomy Observatory) database (https://dept.astro.lsa.umich .edu/datasets/umrao.php), we collected the radio flux densities at $4.8 \mathrm{GHz}, 8 \mathrm{GHz}$, and $14.5 \mathrm{GHz}$ and used the following method to calculate their averaged flux densities and spectral indices, similarly to Yuan \& Fan (2011) [30] and Yuan et al. (2014) [31]. Firstly, at each band $(4.8 \mathrm{GHz}, 8 \mathrm{GHz}$, and 14.5 $\mathrm{GHz}$ ), we average the flux densities within the same bin and obtain $N$ sets of data: $D_{i}\left(J_{i}, F_{4.8 \mid i}, F_{8 \mid i}, F_{14.5 \mid i}\right)(i \in N)$, which represent the flux density at each band. Secondly, based on the relation,

$$
F_{v} \propto v^{-\alpha}
$$

$(\nu=4.8 \mathrm{GHz}, 8 \mathrm{GHz}$ and $14.5 \mathrm{GHz})$, we obtain

$$
\log F_{\nu}=-\alpha \log \nu+C
$$

and use linear fit to calculate the spectral indices $(\alpha)$.

There are 8 blazars $(0235+164,0430+052,1156+295$, 3 C345, 1308+326, 1413+135, 3C454.3, and 1749+096) with known quasi-periodicity and enough $\alpha$ values $(N>50)$. We use them to build a sample, for which, the spectral indices $(\alpha)$ and the averaged flux densities within the respective bins are displayed in Figure 1. The upper panels show the averaged flux densities at $4.8 \mathrm{GHz}, 8 \mathrm{GHz}$, and $14.5 \mathrm{GHz}$, and the lower panels show the spectral indices. The detailed descriptions about $\alpha$ have been listed in Table 1, where Col. 1: Name, Col.
2: redshift $z$, Col. 3: Type, ' $F$ ': FSRQ, ' $B L$ ': BL object, Col. 4: number of $\alpha$ values, Col. 5: $\alpha_{\min }$ (the minimum $\alpha$ ), Col. 6: $\alpha_{\max }($ the maximum $\alpha$ ), and Col. 7: $\bar{\alpha}$ (the averaged $\alpha$ ).

\section{The Relations between the Spectral Indices and Flux Densities}

3.1. Methods. For two variable data sets, $x_{i}, y_{i}(i=1, n)$, we use the linear fitting to analyze their correlations, $y=$ $(k \pm \Delta k) x+\left(k_{0} \pm \Delta k_{0}\right)$, where $k$ is the slope, $k_{0}$ is the intersection, $p$ is the Student's $t$ probability, and $n$ is the number of points in the data set. The Pearson's correlation coefficient $r$ is expressed as [32-34]

$$
r=\frac{\sum\left(x_{i}-\bar{x}\right)\left(y_{i}-\bar{y}\right)}{\sqrt{\sum\left(x_{i}-\bar{x}\right)^{2}} \sqrt{\sum\left(y_{i}-\bar{y}\right)^{2}}},
$$

where $\bar{x}$ is the averaged value of $x_{i}$ and $\bar{y}$ is the averaged value of $y_{i}$.

When $x, y$ show elliptical appearance, we use the following elliptic curve to make the elliptic fit:

$$
a x^{2}+b x y+c y^{2}+d x+e y+f=0,
$$

with $a, b, c, d, e, f$ being free parameters and $|a|$ being normalized.

Considering $x$ as $F_{14.5}$ and $y$ as $\alpha$, we make the following calculations.

3.2. The Linear Correlations. We use the linear correlations to analyze the relations between $F_{14.5}$ and $\alpha$. The results are shown in Figure 2, where the solid lines indicate the fit between $F_{14.5}$ and $\alpha$. The slope $k$, the intersection $k_{0}$, the correlation coefficient $r$, and the chance probability $p$ are listed in Table 2, where Col. 1: Name, Col. 2: the slope $k$, Col. 3: the slope error $\Delta k$, Col. 4: the intersection $k_{0}$, Col. 5: the intersection error $\Delta k_{0}$, Col. 6: the correlation coefficient $r$, and Col. 7: the chance probability $p$. Among the eight target sources, $r$ values are in the range from -0.869 to -0.328 , so $\alpha$ and $F_{14.5}$ show strong anticorrelations.

3.3. The Elliptic Fitting. For every targeted source, based on time sequence, and the variable trend of spectral indices $(\alpha)$ dependent on flux densities, we can find the elliptic appearances in the $\alpha-F_{14.5}$ distributions, as seen in Figure 3, where 

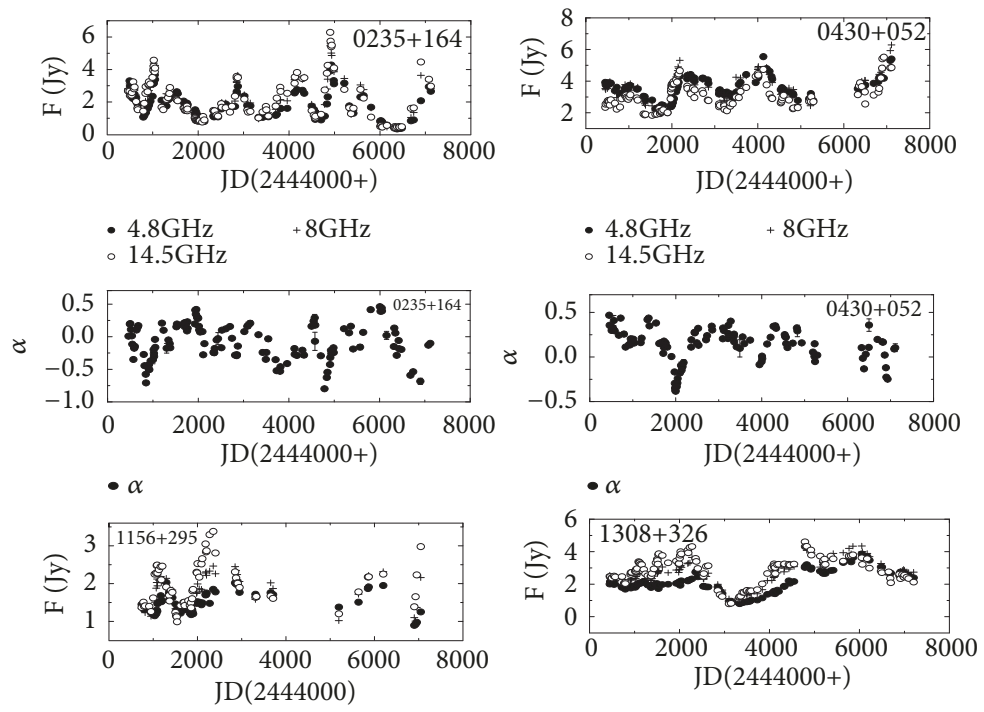

$$
\begin{array}{ll}
-4.8 \mathrm{GHz} & +8 \mathrm{GHz} \\
-14.5 \mathrm{GHz} &
\end{array}
$$

- $4.8 \mathrm{GHz} \quad \circ 14.5 \mathrm{GHz}$ $+8 \mathrm{GHz}$
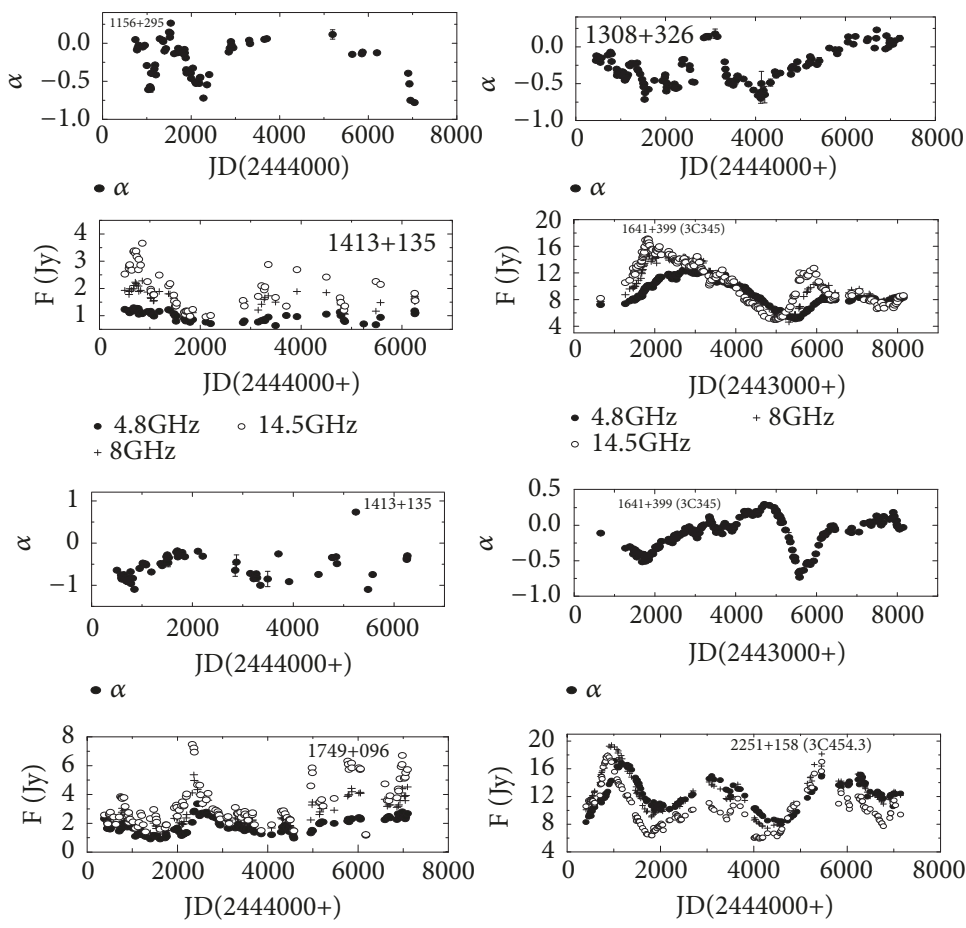

- $4.8 \mathrm{GHz}$ 。 $14.5 \mathrm{GHz}$

- $4.8 \mathrm{GHz}$ 。 $14.5 \mathrm{GHz}$ $+8 \mathrm{GHz}$
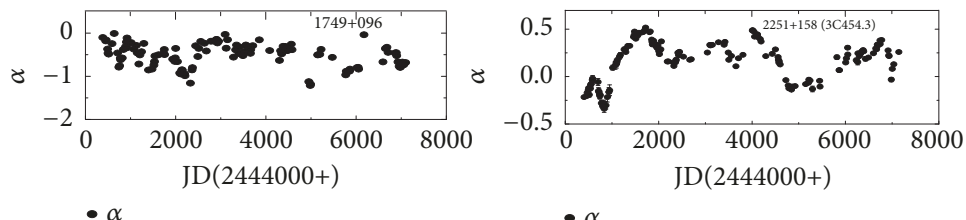

FIGURE 1: The averaged flux densities and spectral variances of the 8 sources, i.e., $0235+164,0430+052,1156+295,1308+326,1413+135,1641+399$ (3C 345), 1749+096, and 2251+158 (3C 454.3) as a function of time measured in JD. The averaged flux densities are shown in the upper panels and the spectral variances are shown in the lower panels, in which the filled circles stand for $4.8 \mathrm{GHz}$, the crosses stand for $8 \mathrm{GHz}$, and the open circles stand for $14.5 \mathrm{GHz}$. 

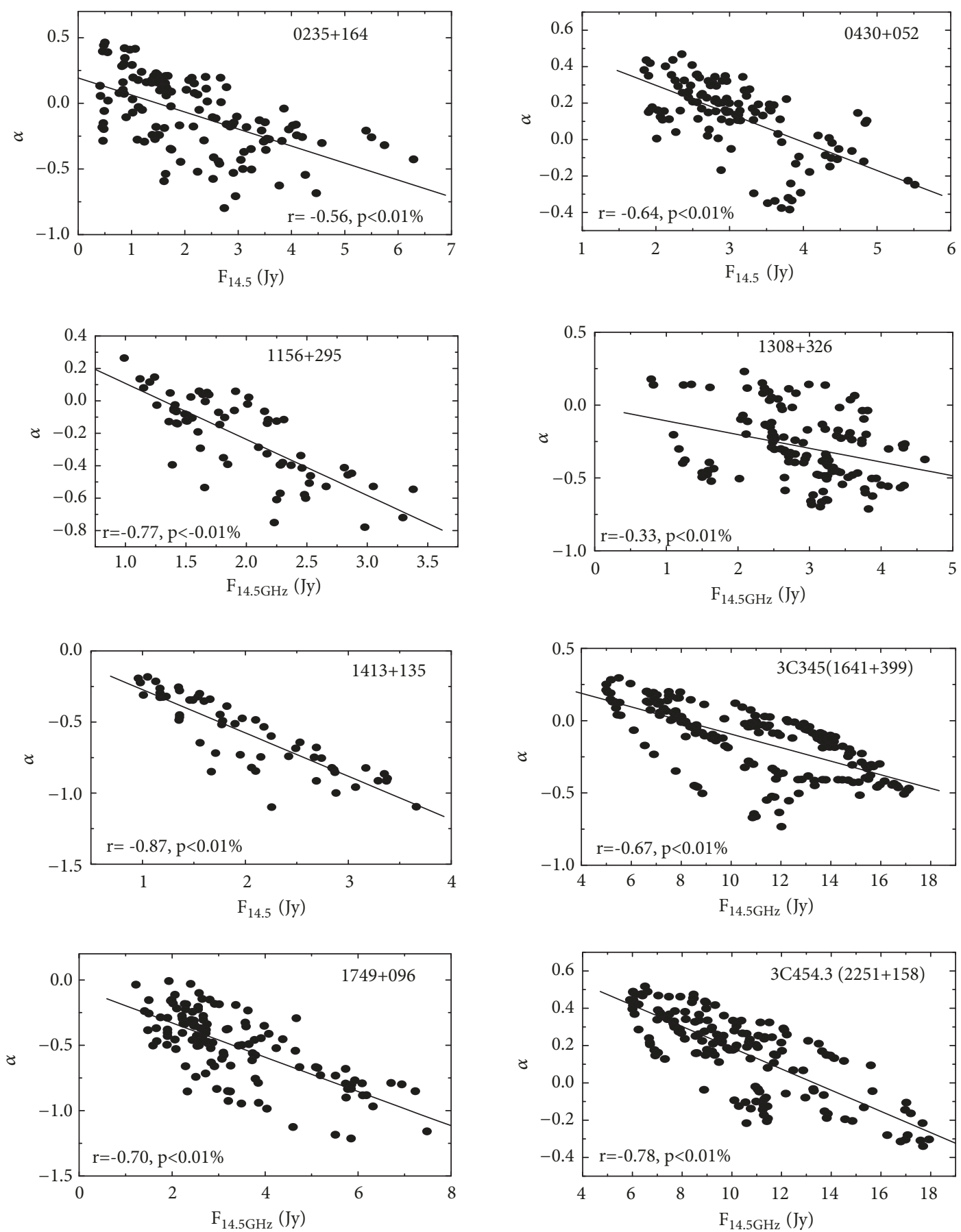

Figure 2: The correlations between the spectral indices $(\alpha)$ and flux densities $\left(F_{14.5}\right)$. The solid lines indicate linear fits between $\alpha$ and $F_{14.5}$.

the filled circles, crosses, and open circles stand for the different elliptic appearances. For the whole elliptic appearances, the variable trends are anticlockwise. We use the elliptic curve to make fit, and the results are shown in Figure 3 and listed in Table 3.

In Table 3,

\section{Col. 1: Name;}

Col. 2: sequence number of elliptic circle;

Col. 3: $T_{B}$, beginning time in Julian Date (JD);
Col. 4: $T_{L}$, end time of elliptic circle in JD;

Col. 5: the time span in yr;

Col. 6-11: the coefficients of elliptic fitting.

\section{Discussion}

Phenomenon that there are cycle structures in blazar variabilities can help us study the theoretical models in both optical and radio light curves. This method has been used in analysis of the quasi-periodic behavior in some blazars 
TABLE 2: The linear correlations between the spectral indices and flux densities.

\begin{tabular}{|c|c|c|c|c|c|c|}
\hline $\begin{array}{l}\text { Name } \\
(1)\end{array}$ & $\begin{array}{c}k \\
(2)\end{array}$ & $\begin{array}{l}\Delta k \\
(3)\end{array}$ & $\begin{array}{l}k 0 \\
(4)\end{array}$ & $\begin{array}{c}\Delta k 0 \\
(5)\end{array}$ & $\begin{array}{c}r \\
(6)\end{array}$ & $\begin{array}{c}p \\
(7)\end{array}$ \\
\hline $0235+164$ & -0.12 & 0.02 & 0.18 & 0.04 & -0.56 & $<0.01 \%$ \\
\hline $0430+052$ & -0.16 & 0.02 & 0.61 & 0.06 & -0.63 & $<0.01 \%$ \\
\hline $1156+295$ & -0.35 & 0.03 & 0.45 & 0.07 & -0.78 & $<0.01 \%$ \\
\hline $1308+326$ & -0.09 & 0.02 & -0.01 & 0.07 & -0.33 & $0.01 \%$ \\
\hline $1413+135$ & -0.30 & 0.02 & 0.03 & 0.05 & -0.87 & $<0.01 \%$ \\
\hline $3 C 345$ & -0.05 & 0.01 & 0.38 & 0.04 & -0.67 & $<0.01 \%$ \\
\hline $1749+096$ & -0.13 & 0.01 & -0.07 & 0.04 & -0.71 & $<0.01 \%$ \\
\hline 3C 454.3 & -0.06 & 0.01 & 0.76 & 0.04 & -0.78 & $<0.01 \%$ \\
\hline
\end{tabular}

TABLE 3: The elliptic fitting of 8 blazars.

\begin{tabular}{lcccccccccc}
\hline $\begin{array}{l}\text { Name } \\
(1)\end{array}$ & Seq. & $T_{B}$ & $T_{L}$ & Span & $a$ & $b$ & $c$ & $d$ & $e$ \\
$(4)$ & $(4)$ & $(5)$ & $(6)$ & $(7)$ & $(8)$ & $\begin{array}{c}f \\
(9)\end{array}$ \\
\hline $0235+164$ & 1 & 2444460.459 & 2446363.609 & 5.21 & 0.055 & 0.242 & 0.867 & -0.226 & -0.332 & 0.159 \\
& 2 & 2446406.692 & 2448333.603 & 5.28 & 0.006 & 0.238 & 0.873 & -0.255 & -0.257 & 0.214 \\
& 3 & 2448499.623 & 2450431.415 & 5.29 & 0.037 & 0.206 & 0.934 & -0.171 & -0.226 & 0.064 \\
\hline $0430+052$ & 1 & 2444452.616 & 2446851.019 & 6.57 & 0.042 & 0.207 & 0.532 & -0.274 & -0.661 & 0.401 \\
& 2 & 2447096.251 & 2449293.781 & 6.02 & -0.024 & -0.132 & -0.664 & 0.182 & 0.629 & -0.336 \\
\hline $1156+295$ & 1 & 2444740.566 & 2445899.332 & 3.17 & -0.14 & -0.404 & -0.412 & 0.439 & 0.585 & -0.336 \\
& 2 & 2445980.910 & 2447706.918 & 4.73 & -0.091 & -0.383 & -0.501 & 0.328 & 0.633 & -0.296 \\
& 3 & 2449194.517 & 2451045.216 & 5.07 & 0.141 & 0.428 & 0.623 & -0.412 & -0.396 & 0.286 \\
\hline $1308+326$ & 1 & 2443753.007 & 2448787.811 & 13.78 & -0.046 & -0.259 & -0.951 & 0.129 & 0.006 & -0.095 \\
& 2 & 2448792.829 & 2451203.896 & 6.6 & -0.033 & -0.223 & -0.577 & 0.209 & 0.685 & -0.319 \\
\hline $1413+135$ & 1 & 2444502.914 & 2447492.380 & 8.18 & -0.091 & -0.479 & -0.857 & 0.064 & -0.132 & -0.084 \\
\hline 3 C 345 & 1 & 2443657.970 & 2447349.477 & 10.11 & 0.003 & 0.075 & 0.659 & -0.068 & -0.668 & 0.33 \\
& 2 & 2447419.046 & 2451142.448 & 10.19 & -0.008 & -0.115 & -0.509 & 0.116 & 0.749 & -0.39 \\
\hline $1749+096$ & 1 & 2444384.487 & 2445934.132 & 4.24 & -0.092 & -0.276 & -0.782 & 0.352 & -0.04 & -0.421 \\
& 2 & 2445980.169 & 2447567.092 & 4.35 & -0.044 & -0.217 & -0.847 & 0.224 & 0.034 & -0.394 \\
& 3 & 2447655.821 & 2451095.560 & 9.42 & 0.033 & 0.285 & 0.932 & -0.079 & 0.137 & 0.155 \\
\hline 3 C 454.3 & 1 & 2444543.911 & 2447805.613 & 8.93 & -0.002 & -0.057 & -0.465 & 0.063 & 0.782 & -0.406 \\
& 2 & 2448006.686 & 2451143.235 & 8.59 & -0.002 & -0.059 & -0.599 & 0.055 & 0.736 & -0.304 \\
\hline
\end{tabular}

$[17,18,35,36]$. Villata \& Raiteri (1999) [37] pointed out that the quasi-periodic behavior of Mkn501 can be associated with the helical structures of the jet. Pyatunina et al. (2006) [38] presented that the activity of blazars can be characterized by two parameters: the time scale for the activity of the central engine, $T_{a c t}$, and the time scale for the evolution of the jet, $T_{\text {evo }}$. They also proposed that $T_{a c t}$ determines an 'activity cycle' for the source. Pyatunina (2007) [17] defined the activity cycles as the time intervals between the successive 'core' outbursts. They obtained cycle times of more than 14 yr for $1308+326$, about $12 \mathrm{yr}$ for 2223-052, and $12.4 \mathrm{yr}$ for $2251+158$ (3C 454.3). Our circle durations are 6.6 years and 13.78 years for $1308+326$, which are consistent with Pyatunina (2007) [17]. For 2251+158, our results show that 8.93 years is shorter than Pyatunina (2007) [17]. Main reason for this should be that we averaged the light curves with the fixed time intervals and then lead to the less observations.

So, studies about the activity cycles are very important for testing the theoretical models, the jet structure, the properties of central engine, and predicting the likely outbursts.
According to the time interval calculated by the elliptic fitting, we can divide the total light curves into different parts, as seen in Figure 4, and then compare the time intervals of elliptic circle with the quasi-periodicities calculated by the averaged light curves and spectral variances.

The most common tool for periodicity analysis of both evenly and unevenly sampled signals is the periodogram method, which is an estimator of the signal energy in the frequency domain [39]. Lomb (1976) [40] introduced a modified form of this method.

We used the Lomb's periodogram method to calculate the periodicity and used the half width at half maximum (HWHM) to calculate the corresponding error. For every targeted sources, based on the averaged light curves and spectral indices, we calculate the two periodic signals, then place them together, and choose the common signal as the last periodicity, $P_{\alpha \bar{F}}$. The period signals are displayed in Figure 5, where the upper panels show the averaged flux density, and the lower panels show the spectral variances. The red noise [41] with the noise levels $80 \%, 90 \%, 95 \%$, and $99 \%$ is also shown in Figure 5. 

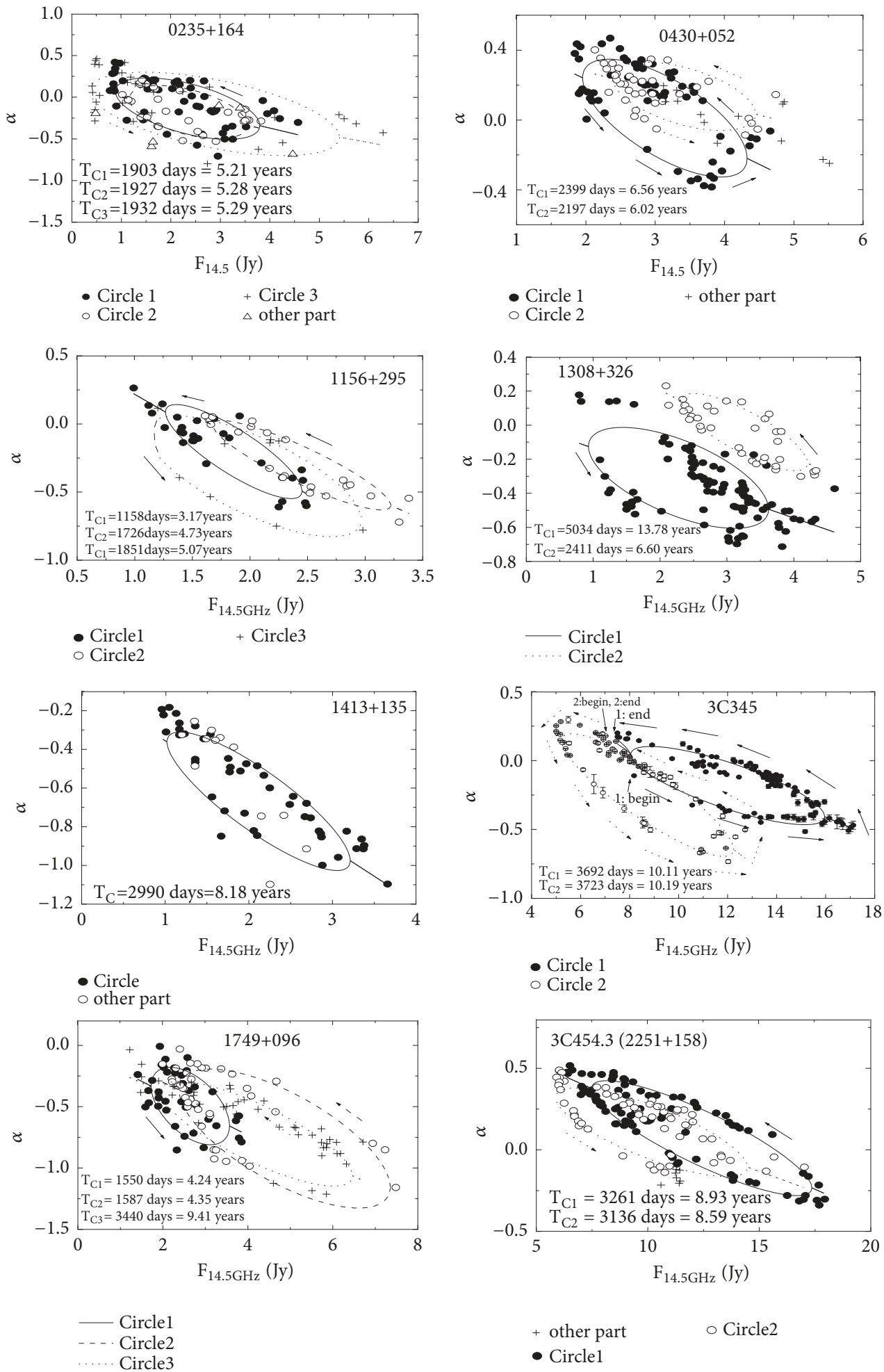

Figure 3: The $\alpha-F_{14.5}$ circles of the target sources. The elliptic solid lines, dotted lines, and dashed lines indicate the elliptic fit results based on the time sequences. 

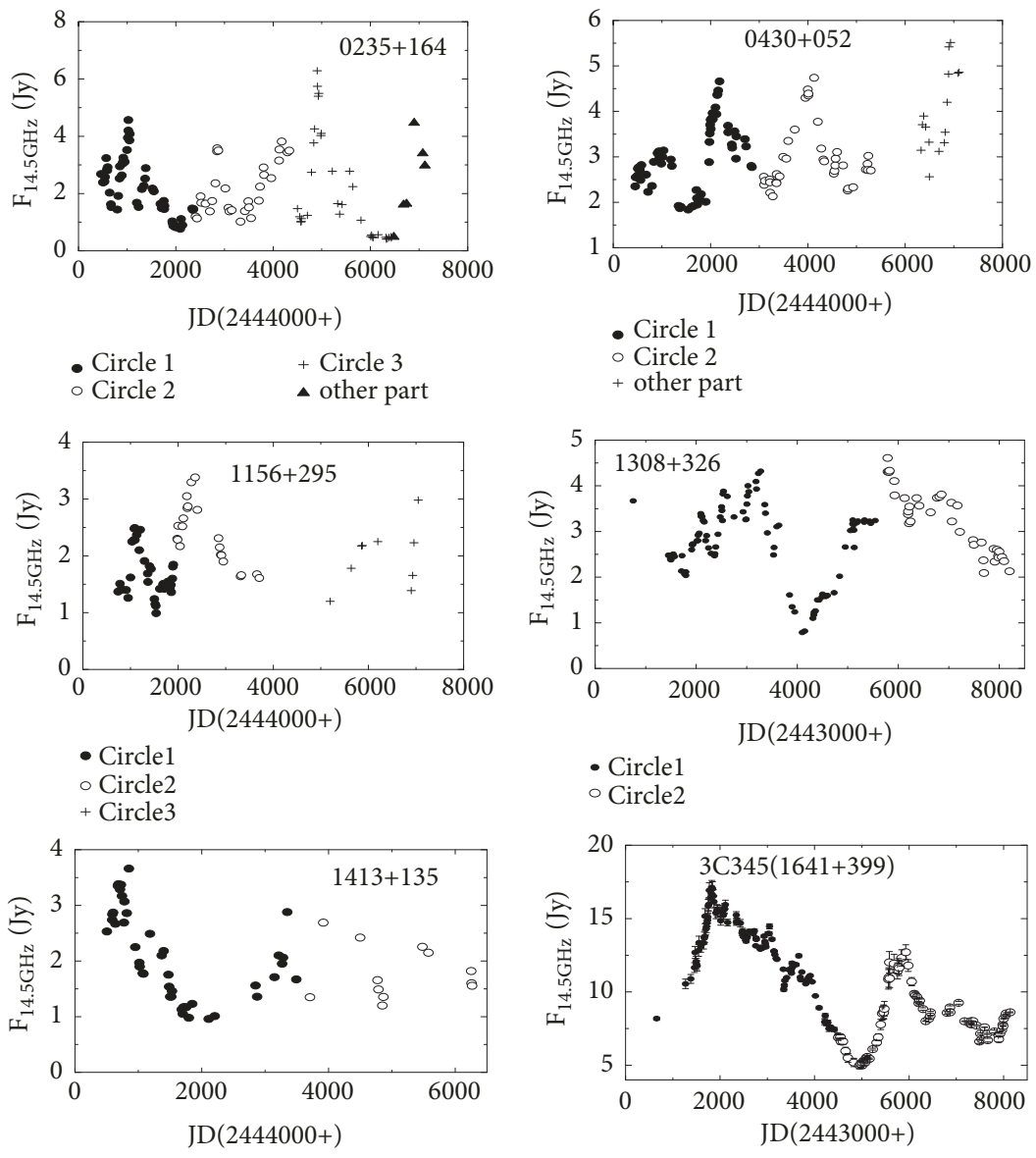

$$
\begin{aligned}
& \text { - Circle } \\
& \text { o other part }
\end{aligned}
$$

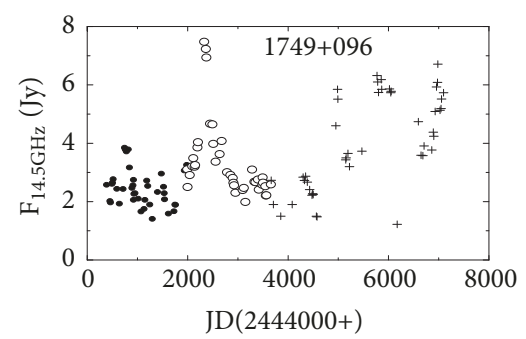

- Circle1
- Circle2
+ Circle3

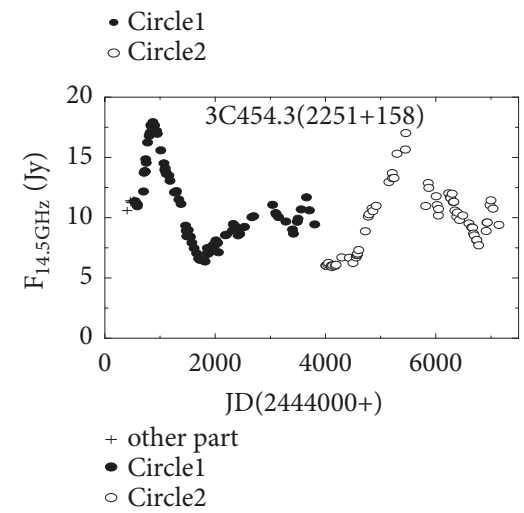

FIGURE 4: For each source, based on the time interval of elliptic circles, the light curves can be divided into different parts.

For every targeted source, the time interval of elliptic circle $\left(T_{c}\right)$, the periodicities calculated from the averaged light curves, and spectral variances $\left(P_{\alpha \bar{F}}\right)$ are listed in Table 4 . In order to make comparison, we quoted the quasi-periodicities $\left(Q_{F}\right)$ calculated by Fan, Liu \& Yuan (2007) [14], which were calculated from the whole light curves and listed in Table 4.

We use a linear fitting to compare $T_{c}, P_{\alpha \bar{F}}$, and $Q_{F}$. The results show that $P_{\alpha \bar{F}}=(0.90 \pm 0.52) T_{c}-(0.88 \pm 3.63)$, with $r=0.58, p=13.3 \% ; Q_{F}=(0.85 \pm 0.27) T_{c}+(0.63 \pm 1.92)$, with $r=$ $0.78, p=2.1 \%$, as seen in Figure 6, with the black line standing for $T_{c}$ vs. $P_{\alpha \bar{F}}$ and the red line standing for $T_{c}$ vs. $Q_{F}$. The two fitting results $T_{c}$ vs. $P_{\alpha \bar{F}}$ and $T_{c}$ vs. $Q_{F}$ can be consistent with each other, which show strong correlations between the time internal of elliptic circle and quasi-periodicities not only calculated from the whole light curve $Q_{F}$, but also calculated from the averaged light curves and spectral variances $\left(P_{\alpha \bar{F}}\right)$.

Quasi-periodicity is a hot research topic in radio variability of blazars, but the reason for the periodicity is unclear. There are many models proposed to explain this phenomenon, for example, the binary black-hole model, the thermal instability model, and the perturbation model [14]. Many authors [42-45] apply the model of a moving shock in a relativistic jet with a helical magnetic field to explain the flux 

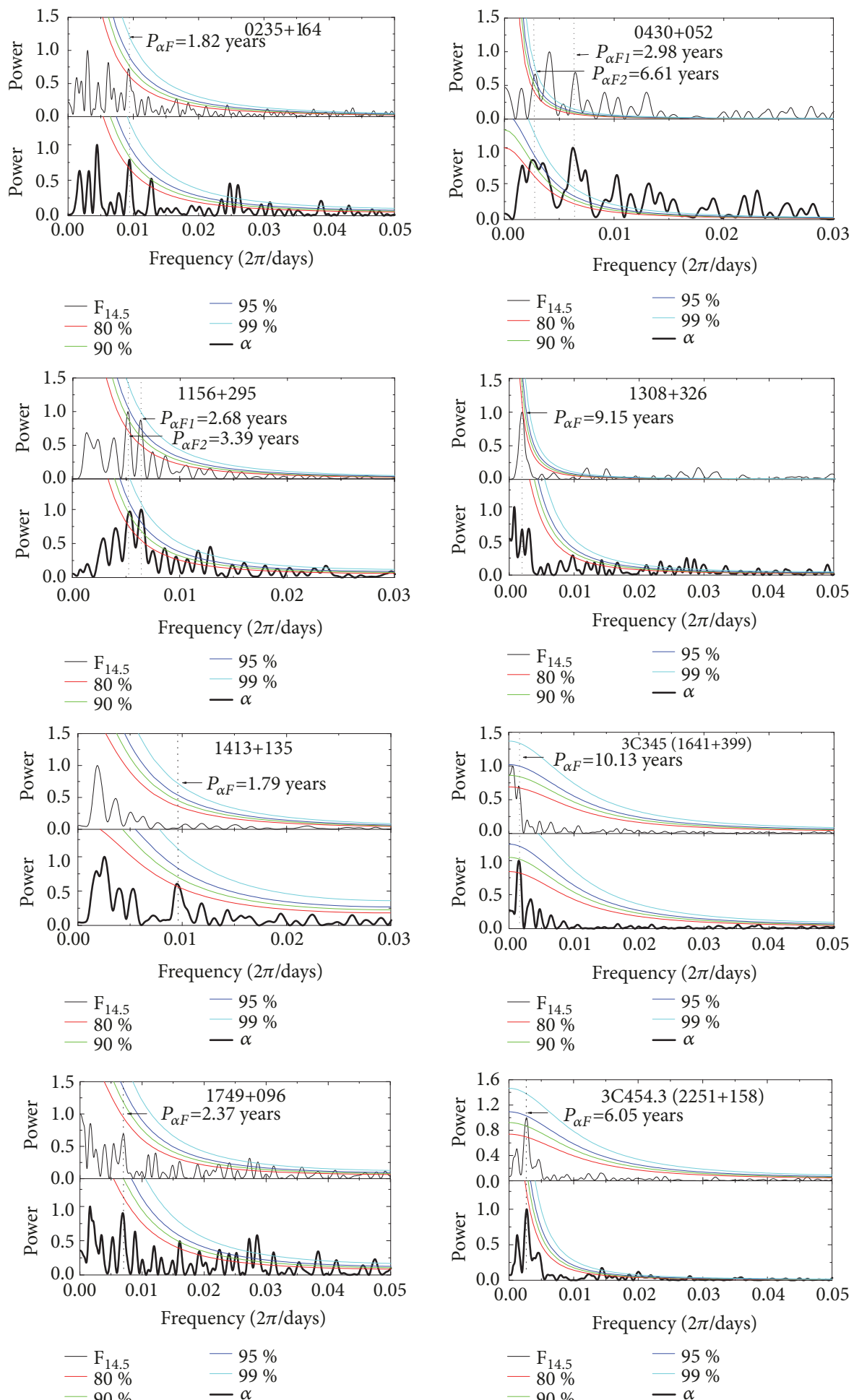

Figure 5: The quasi-periodicities of 8 targeted sources. The upper panels are derived from the light curves and the lower panels are from spectral indices. The black lines indicate periodic signals. The red, green, blue, and cyan lines show $80 \%, 90 \%$, 95\%, and $99 \%$ red noise level, respectively. 
TABLE 4: The time spans and long-term quasi-periodicities of 8 blazars.

\begin{tabular}{lccc}
\hline Name & $T_{c}$ & $P_{\alpha \bar{F}}$ & $Q_{F}$ \\
& $/ \mathrm{yr}$ & $1 \mathrm{yr}$ & $5.7 \pm 0.3,10.0 \pm 1.3$ \\
\hline $0235+164$ & $5.21,5.28,5.29$ & $1.82 \pm 0.57$ & $4.3 \pm 0.2,11.8 \pm 1.1,13.6 \pm 1.2$ \\
$0430+052$ & $6.56,6.02$ & $2.98 \pm 0.07,6.61 \pm 0.84$ & $3.3 \pm 0.1,3.4 \pm 0.1,10.4 \pm 1.2$ \\
$1156+295$ & $3.17,4.73,5.07$ & $2.68 \pm 0.15,3.39 \pm 0.16$ & $5.9 \pm 0.4,11.8 \pm 1.1$ \\
$1308+326$ & $6.60,13.78$ & $9.15 \pm 1.75$ & $8.2 \pm 0.4$ \\
$1413+135$ & 8.18 & $1.79 \pm 0.06$ & $10.9 \pm 0.7,11.3 \pm 0.4$ \\
3 C 345 & $10.11,10.19$ & $10.13 \pm 1.08$ & $6.3 \pm 0.4,9.0 \pm 0.9$ \\
$1749+096$ & $4.24,4.35,9.41$ & $2.37 \pm 0.36$ & $6.3 \pm 0.2,11.8 \pm 1.1$ \\
3C 454.3 & $8.93,8.59$ & $6.05 \pm 1.06$ &
\end{tabular}

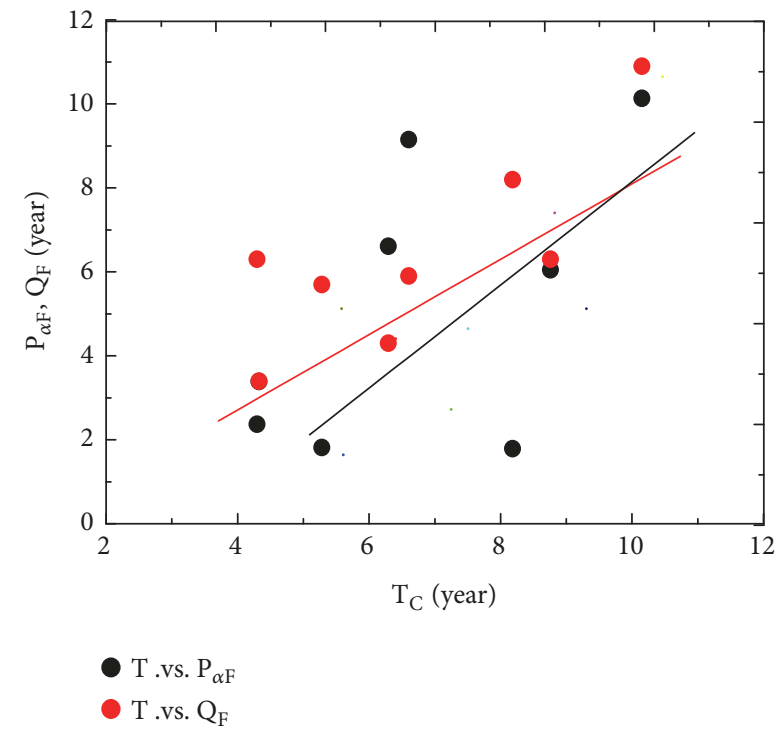

Figure 6: Comparison between $T_{c}$ and $P_{\alpha \bar{F}}, Q_{F}$. The black line stands for $T_{c}$ vs. $P_{\alpha \bar{F}}$, and the red line stands for $T_{c}$ vs. $Q_{F}$.

density variances. The $\alpha-F_{14.5}$ circles might come from the helical jet produced by the binary black holes.

\section{Conclusion}

In this paper, we choose eight blazars from the UMRAO data base to calculate the spectral indices. We average the light curves with the fixed interval, calculate the averaged flux densities $(\bar{F})$ and spectral indices $(\alpha)$, and then compare the relations between $\alpha$ and $\bar{F}$.

For each source, we obtain strong anticorrelations between $\alpha$ and $F_{14.5}$, which can combine the emission properties and radio variabilities.

Based on time sequence, the $\alpha-F_{14.5}$ distributions showed elliptic appearances and the variable trends are anticlockwise. We use the elliptic curve to make fit, find that the variation directions of elliptic cycle are anticlockwise, and then obtain the cycle durations. Based on the averaged light curves and spectral variances, we calculate the quasi-periodicities and compare them with the cycle durations. The results show that they are consistent with each other, so the elliptic appearances should come from the helical structures of jets or the jet models containing at least two emitting regions.

\section{Data Availability}

The text formatting data used to support the findings of this study come from the University of Michigan Radio Observatory (UMRAO), which are public. The main results have been listed in Tables 1-3, which can be used by others.

\section{Conflicts of Interest}

The author declares that they have no conflicts of interest.

\section{Acknowledgments}

The work is partially supported by the National Natural Science Foundation of China (NSFC U1831119, NSFC 11733001, NSFC U1531245, NSFC 10633010, NSFC 11173009, and NSFC U1431112), Natural Science Foundation of Guangdong Province (2017A030313011), supports for Astrophysics Key Subjects of Guangdong Province and Guangzhou City, and Science and Technology Program of Guangzhou (201707010401). This research has made use of data from the University of Michigan Radio Astronomy Observatory which has been supported by the University of Michigan and the National Science Foundation.

\section{References}

[1] C. M. Urry and P. Padovani, "Unified schemes for radio-loud active galactic nuclei," Publications of the Astronomical Society of the Pacific, vol. 107, p. 803, 1995.

[2] U. Marie-Helene, L. Maraschi, and C. M. Urry, "Variability of active galactic nuclei," Annual Review of Astronomy and Astrophysics, vol. 35, pp. 445-502, 1997.

[3] M. Stickel, J. W. Fried, and H. Kuhr, "The complete sample of 1 Jy BL Lac objects. II - Observational data," Astronomy and Astrophysics Supplement Series, vol. 98, pp. 393-442, 1993.

[4] G. Ghisellini, F. Tavecchio, L. Foschini, and G. Ghirlanda, "The transition between BL Lac objects and flat spectrum radio quasars," Monthly Notices of the Royal Astronomical Society, vol. 414, no. 3, pp. 2674-2689, 2011.

[5] T. Sbarrato, G. Ghisellini, L. Maraschi, and M. Colpi, "The relation between broad lines and $\gamma$-ray luminosities in Fermi 
blazars," Monthly Notices of the Royal Astronomical Society, vol. 421, no. 2, pp. 1764-1778, 2012.

[6] G. Ghisellini and F. Tavecchio, "Fermi/LAT broad emission line blazars," Monthly Notices of the Royal Astronomical Society, vol. 448, pp. 1060-1077, 2015.

[7] W. E. Kunkel, "A harmonic analysis of the light variation of 3C273," The Astronomical Journal, vol. 72, p. 1341, 1967.

[8] A. Sillanpaa, S. Haarala, M. J. Valtonen, B. Sundelius, and G. G. Byrd, "OJ 287 - Binary pair of supermassive black holes," The Astrophysical Journal, vol. 325, pp. 628-634, 1988.

[9] V. E. Chertoprud, L. I. Gudzenko, and L. M. Ozernoy, "ON the character of optical variability of the quasar 3C 273," The Astrophysical Journal, vol. 182, p. 53, 1973.

[10] F. K. Liu, G. Z. Xie, and J. M. Bai, "A historical light curve of ON 231 and its periodic analysis," Astronomy \& Astrophysics, vol. 295, pp. 1-10, 1995.

[11] J. H. Fan, G. Z. Xie, E. Pecontal, A. Pecontal, and Y. Copin, "Historic light curve and long-term optical variation of BL lacertae 2200+420," The Astrophysical Journal, vol. 507, no. 1, pp. 173-178, 1998.

[12] J. H. Fan and R. G. Lin, "The variability analysis of PKS 2155304," Astronomy \& Astrophysics, vol. 355, pp. 880-884, 2000.

[13] G. Z. Xie, S. B. Zhou, B. Z. Dai et al., "Photometric monitoring of 12 BL Lacertae objects," Monthly Notices of the Royal Astronomical Society, vol. 329, no. 4, pp. 689-699, 2002.

[14] J. H. Fan, Y. Liu, and Y. H. Yuan, "Radio variability properties for radio sources," Astronomy \& Astrophysics, vol. 462, pp. 547-552, 2007.

[15] J. H. Fan, O. Kurtanidze, Y. Liu, G. M. Richter, R. Chanishvili, and Y. H. Yuan, "Optical monitoring of two brightest nearby quasars, PHL 1811 and 3C 273," The Astrophysical Journal Supplement Series, vol. 213, no. 2, p. 26, 2014.

[16] Y. Yu-Hai, Jun-hui; F., T. Jun et al., "Optical monitoring of BL Lac object S5 0716+714 and FSRQ 3C 273 from 2000 to 2014," Astronomy \& Astrophysics, vol. 605A, p. 43, 2017.

[17] T. B. Pyatunina, N. A. Kudryavtseva, D. C. Gabuzda et al., "Frequency-dependent time delays for strong outbursts in selected blazars from the Metsähovi and UMRAO monitoring data bases - II," Monthly Notices of the Royal Astronomical Society, vol. 381, no. 2, pp. 797-808, 2007.

[18] C. M. Raiteri, M. Villata, H. D. Aller et al., "Optical and radio variability of the BL Lacertae object AO 0235+16: A possible 5-6 year periodicity," Astronomy \& Astrophysics, vol. 377, pp. 396412, 2001.

[19] R. A. Edelson, J. H. Krolik, and G. F. Pike, "Broad-band properties of the CfA Seyfert galaxies. III - Ultraviolet variability," The Astrophysical Journal, vol. 359, pp. 86-97, 1990.

[20] D. Trevese and F. Vagnetti, "Quasar spectral slope variability in the optical band," The Astrophysical Journal, vol. 564, pp. 624630, 2002.

[21] F. Vagnetti, D. Trevese, and R. Nesci, "Spectral slope variability of BL Lacertae objects in the optical band," The Astrophysical Journal, vol. 590, no. 1 I, pp. 123-127, 2003.

[22] A. Wierzcholska, M. Ostrowski, A. Stawarz, S. Wagner, and M. Hauser, "Longterm optical monitoring of bright BL Lacertae objects with ATOM: Spectral variability and multiwavelength correlations," Astronomy \& Astrophysics, vol. 573, p. 69, 2015.

[23] M. I. Carnerero, C. M. Raiteri, M. Villata, J. Acosta Pulido, P. Smith, and V. Larionov, "Dissecting the long-term emission behaviour of the BL Lac object Mrk 421," Monthly Notices of the Royal Astronomical Society, vol. 472, pp. 3789-3804, 2017.
[24] J. C. Isler, C. M. Urry, P. Coppi et al., "A consolidated framework of the color variability in blazars: long-term optical/nearinfrared observations of 3C 279," The Astrophysical Journal, vol. 844, no. 2, p. 107, 2017.

[25] J. A. Stevens and W. K. Gear, "Variations in the broad-band spectra of BL Lac objects: millimetre observations of an Xray-selected sample," Monthly Notices of the Royal Astronomical Society, vol. 307, pp. 403-412, 1999.

[26] D. Trevese, R. D. Kron, and A. Bunone, "Continuum variability of active galactic nuclei in the optical-ultraviolet range," The Astrophysical Journal, vol. 551, pp. 103-110, 2001.

[27] B. Z. Dai, X. H. Li, Z. M. Liu et al., "The long-term multiband optical observations and colour index for the quasar 3C 273," Monthly Notices of the Royal Astronomical Society, vol. 392, no. 3, pp. 1181-1192, 2009.

[28] P. P. B. Beaklini and Z. Abraham, "3C 273 variability at $7 \mathrm{~mm}$ : evidence of shocks and precession in the jet," Monthly Notices of the Royal Astronomical Society, vol. 437, pp. 489-496, 2014.

[29] Y. H. Yuan and J. H. Fan, "The relationship between the spectrum and flux density of 3C273 and 3C446," Astrophysics and Space Science, vol. 357, p. 123, 2015.

[30] Y. H. Yuan and J. H. Fan, "Long term periodicity analysis of the spectral index of 2251+158," Research in Astronomy and Astrophysics, vol. 11, pp. 286-292, 2011.

[31] Y. Yuan, "Relation between radio polarization and spectral index of blazars," International Journal of Astronomy and Astrophysics, vol. 35, no. 3, pp. 417-421, 2014.

[32] W. H. Press, S. A. Teukolsky, W. T. Vetterling, and B. P. Flannery, Numerical Recipes in Fortran, The art of Scientific Computing, Cambridge University Press, 2nd edition, 1994.

[33] V. Pavlidou, J. L. Richards, W. Max-Moerbeck et al., "Assessing the significance of apparent correlations between radio and gamma-ray blazar fluxes," The Astrophysical Journal, vol. 751, no. 2, p. 149, 2012.

[34] J. Fan, J. H. Yang, J. Zhang et al., "Beaming effect in fermi blazars," Publications of the Astronomical Society of Japan, vol. 65, no. 2, p. 25, 2013.

[35] M. R. Kidger, “The 11 year period in OJ 287 revisited: is it a true long-enduring period?” The Astronomical Journal, vol. 119, no. 5, pp. 2053-2059, 2000.

[36] T. Pursimo, L. O. Takalo, A. Sillanpaa et al., "Intensive monitoring of OJ 287," Astronomy and Astrophysics Supplement Series, vol. 146, pp. 141-155, 2000.

[37] M. Villata and C. M. Raiteri, "Helical jets in blazars," Astronomy \& Astrophysics, vol. 347, pp. 30-36, 1999.

[38] T. B. Pyatunina, N. A. Kudryavtseva, D. C. Gabuzda et al., "Frequency-dependent time-delays for strong outbursts in selected blazars from the Metsähovi and the University of Michigan Radio Astronomy Observatory monitoring databases - I," Monthly Notices of the Royal Astronomical Society, vol. 373, no. 4, pp. 1470-1482, 2006.

[39] T. J. Deeming, "Fourier analysis with unequally-spaced data," Astrophysics and Space Science, vol. 36, pp. 137-156, 1975.

[40] N. R. Lomb, "Least-squares frequency analysis of unequally spaced data," Astrophysics and Space Science, vol. 39, no. 2, pp. 447-462, 1976.

[41] M. Schulz and M. Mudelsee, "REDFIT: Estimating red-noise spectra directly from unevenly spaced paleoclimatic time series," Computers \& Geosciences, vol. 28, no. 3, pp. 421-426, 2002. 
[42] W. Webb, M. Malkan, G. Schmidt, and C. Impey, "The wavelength dependence of polarization of active galaxies and quasars," The Astrophysical Journal, vol. 419, p. 494, 1993.

[43] K. S. Cheng, J. H. Fan, and L. Zhang, "Basic properties of gamma-ray loud blazars," Astronomy \& Astrophysics, vol. 352, pp. 32-38, 1999.

[44] N. Kawakatu, M. Imanishi, and T. Nagao, "Anticorrelation between the mass of a supermassive black hole and the mass accretion rate in type 1 ultraluminous infrared galaxies and nearby QSOs," The Astrophysical Journal, vol. 661, no. 2 I, pp. 660-671, 2007.

[45] M. F. Gu, Z. Y. Chen, and X. W. Cao, "MBH- $\sigma$ relation in Sloan Digital Sky Survey flat-spectrum radio quasars," Monthly Notices of the Royal Astronomical Society, vol. 397, pp. 1705-1710, 2009. 

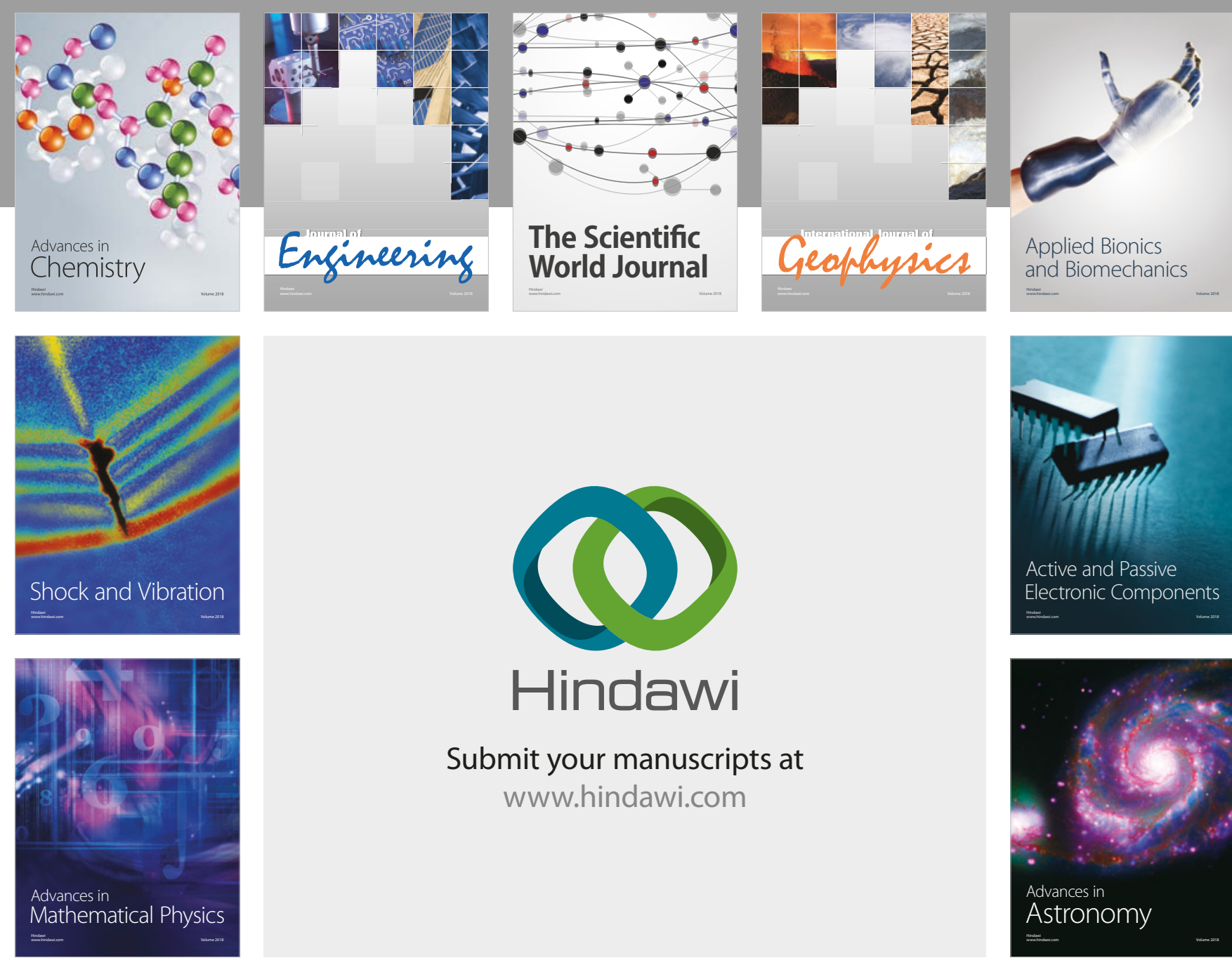

Submit your manuscripts at

www.hindawi.com

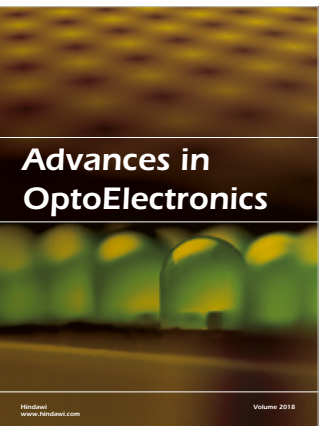

\section{Rotcting Machinery}
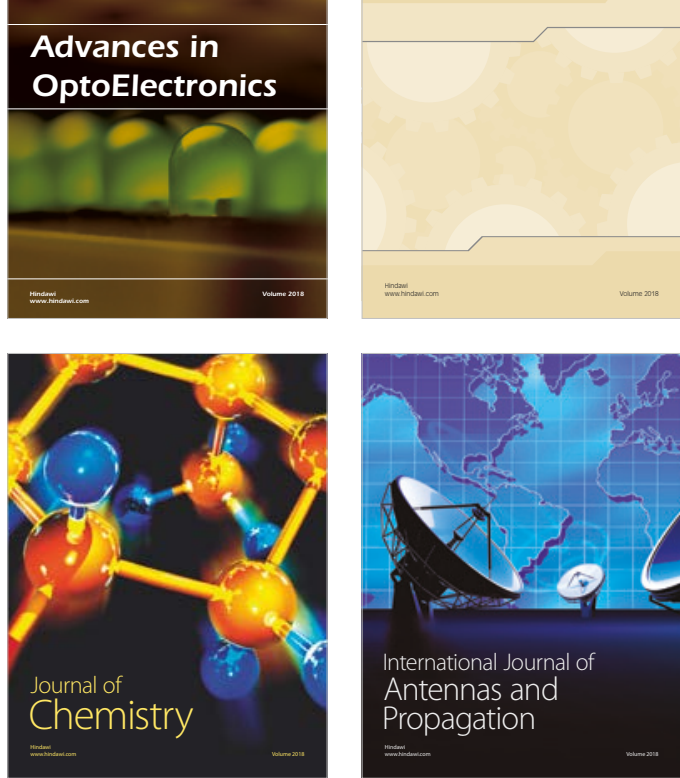

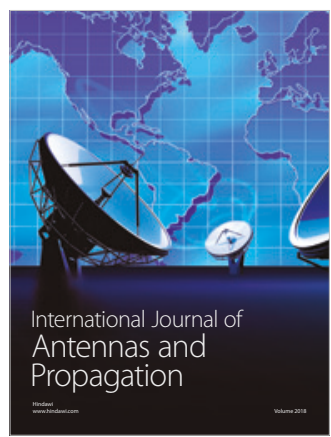

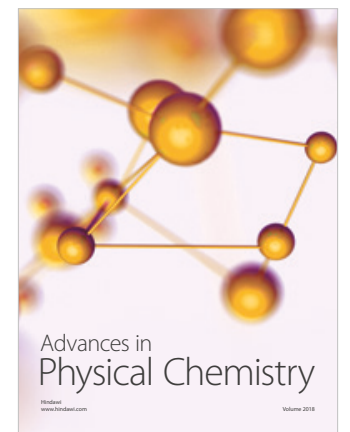

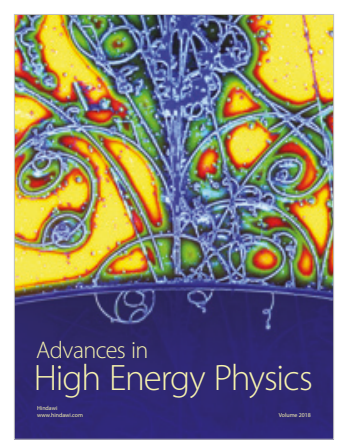

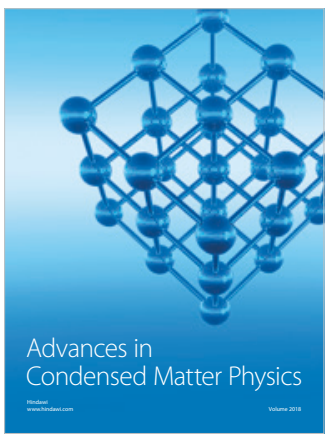

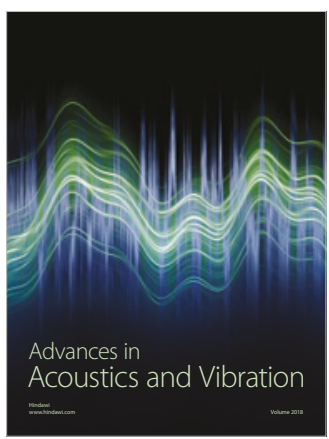

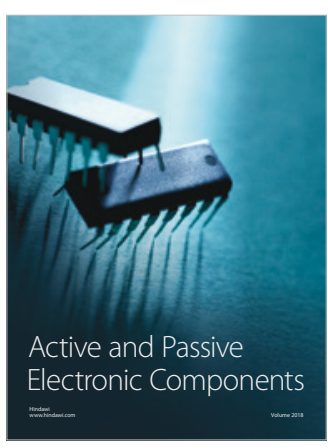
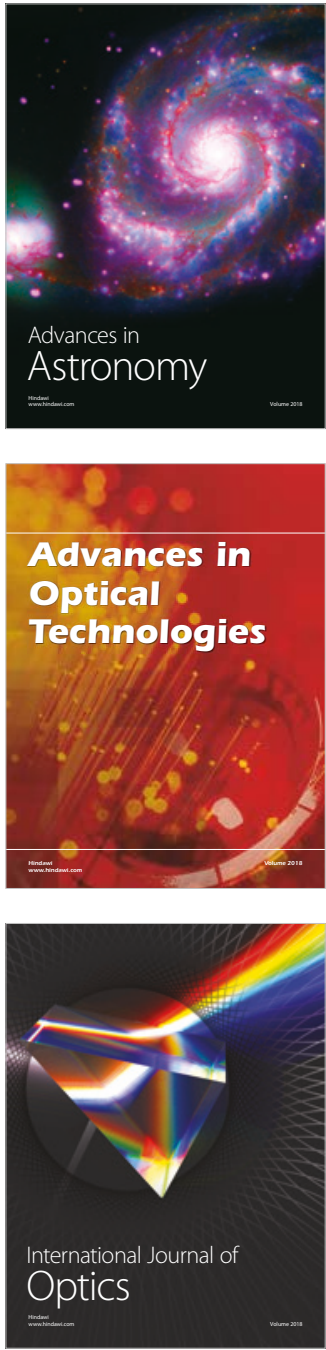